\title{
Derrida und Saussure aus der hermeneutischen Perspektive als Ausgangspunkt für die Schlichtung eines diskurssemantischen Streites
}

\author{
Derrida and Saussure from the Hermeneutical Point of View \\ as a Starting Point for the Settlement of a Discourse-Semantic \\ Dispute
}

Michal Rubáš

\begin{abstract}
On the occasion of a discourse-semantic confusion in the domain of the discourse analysis based on Foucault, the article focuses on Manfred Frank's hermeneutic reception of a neostructuralist key concept, the restance non présente of Jacques Derrida, and inspects both the (neo)structuralist and the hermeneutical theory of sign with regard to their potential to reconcile the phenomenon of language change with a plausible explanation of the ability of the speakers to refer to an identical item in the discourse(s). The text expresses some doubts concerning Frank's theory of sign, which has been formulated in the opposition towards the neostructuralism and in accordance with the "authentic" Saussure, and, on the other hand, appreciates the promising direction of Frank's latest thought grounded in his consciousness theory.
\end{abstract}

\section{Keywords}

Derrida, Frank, Saussure, criticism of the neostructuralist sign theory, the hermeneutic sign theory

Dieser Beitrag entstand im Rahmen des von der Philosophischen Fakultät der Palacký-Universität Olomouc geförderten Projekts ,Hermeneutische Linguistik'/'Hermeneutická lingvistika' (IGA_FF_2016_020). 
Der Anlass zu diesem Text kam aus einer gedankenreichen Auseinandersetzung, die zwei Stammvertreter der auf Foucault sich stützenden linguistischen Diskursanalyse, Dietrich Busse und Wolfgang Teubert, auf den Seiten des von ihnen herausgegebenen programmatischen Sammelbandes Linguistische Diskursanalyse: Neue Perspektiven (Busse, Teubert 2013) miteinander führen. Teubert bekennt sich dabei ausdrücklich zu Derridas Bedeutungstheorie, und Busse sieht darin eine Naivität. ${ }^{1}$

Teubert hält es für unmöglich, dass der Diskursanalytiker das von Diskurs generierte „Gesagte überschreite/t/“, während Busse es bezweifelt, dass sich der Sinn des Gesagten von selbst, ohne Interpretation, ergibt. Busse verteidigt Dikursanalytiker und selbst einige Kognitivisten, indem er hervorhebt, dass diese die Sprachebene nicht verlassen und kein „nicht-sprachliches Wissen“, wie sich Teubert äußert ${ }^{2}$, zum Gegenstand der Diskursanalyse machen. Was in der Diskursanalyse Busse zufolge „zu eruieren“ ist, ist „jetzt unbewusst prägend“; aber „ursprünglich war es bewusst explizit“, es ist anderswo explizit vorgekommen, „von einem Menschen“ explizit gemacht, wenn auch „nicht in diesem Diskurs“ (Busse 2013, S. 169-170).

Auf diese Weise entstehen einige Fragen, wie diese: Was bedeutet „Diskursprodukt“, das nach vielen Diskursanalytiker der Grundcharakter von Bedeutungen (und nach einigen wie Teubert sogar von Denksubjekten) sein soll, wenn in verschiedenen Diskursen dieselben Bedeutungselemente (einmal implizit, andermal explizit) vorkämen? Folgt

1 Ich habe anderswo zu zeigen versucht, dass die Unstimmigkeit zwischen beiden Denkern im Keime ihres linguistischen Unternehmens schon von Anfang an enthalten war, nämlich in Foucaults Diskursbegriff. Durch Teuberts Derridianismus wird jede kritische und wissenschaftliche Reflexion in ihrer Möglichkeit ernst bedroht, durch Busses Hermeneutik werden die neostrukturalistischen Reden über sinnerzeugende Kraft der „Diskurse“ und aller überindividuellen Strukturen unterminiert und der Foucaultsche diskursanalytische Boden verlassen. Vgl. den Sammelband Kotůlková, V., Rykalová, G. (Hg.) Zentrum und Peripherie (Opava 2016), das diesjährlich erscheint.

2 Teuberts Position ist die folgende: Diskurs ist autopoietisch, selbst-referentiell, unergründlich und unvorhersehbar. Er bewegt sich von selbst, indem seine Momente permutieren, sich rekombinieren und variieren (Teubert 2013, S. 55). Erleben ohne Sprache sowie diskursiv unvermittelte Wirklichkeit bedeuten nichts. „Alles /bedeutsame/ ist Diskurskonstrukt“ und „ob er mit externer Realität etwas zu tun hat, lässt sich nicht überprüfen“. Nur das Gesagte hat einen Sinn und nur auf Gesagtes kann man sich beziehen (Teubert 2013, S. 56). Für Teubert ist Text eine „einzigartige Kombination von rekurrenten Elementen“, deren Bedeutung darin bestehe, „was über sie in einzelnen Kontexten gesagt ist“, und dieses Gesagte nennt er „periphrastischen Gehalt“. Für die Bedeutungen gibt es „keinen gemeinsamen Nenner“, und Bedeutung ist ein „Ensemble aller Aussagen“ oder Periphrasen. Bedeutungen seien nicht auf die innovative Initiative der Individuen zurückzuführen, da es nicht „sinnvoll“ ist, über „das solitäre Individuum / .../ zu reden, das sich mit seiner Umwelt kognitiv auseinandersetzt“ (Teubert, 2013, S. 59), und weil Interpretieren „ein kollaborativer Akt“ ist (Teubert, 2013, S. 72). Weil „nicht-sprachliches Vorverständnis unbeschreibbar ist“ und weil es „widersinnig,, und „unmöglich“ ist, vorsprachliche oder sprachlose epistemische Voraussetzungen und unbewusstes, nicht-explizites Wissen freizulegen, muss der kognitive Ansatz (mitsamt Phänomenologie) abgelehnt werden: „Um zu beschreiben, wie sich vorsprachliches Erleben in sprachliche, sozial konventionalisierte Repräsentation übersetzt, müssten wir vorsprachliches Wissen sprachlich repräsentieren können“, und das sei unmöglich. „Individueller Geist“ ist kein einer naturwissenschaftlichen Methode zugängliches Objekt und „der Blick in den Kopf erübrigt sich“ ohnehin. Was „verstehen“ heißt, ist „unergründlich“, wir wissen nur, was darüber gesagt wird. Anderes Beispiel: Was man beim Schwimmen gelernt hat, wie man sich anfühlt, lässt sich nicht in Worte fassen (Teubert 2013, S. 58). Der Sprachforscher soll das Gesagte so aufbereiten, damit der Diskursausschnitt zugänglich wird, der für die Klärung einer Sinnfrage relevant ist. Diese Rekontextualisierung des Sinnes hat Teubert wahrscheinlich im Sinn, wenn er Gadamers Hermeneutik ablehnt mit der Begründung, dass Gadamer die Analyse nicht von der Interpretation trennt (Teubert 2013, S. 73). 
nicht daraus, dass nicht der Diskurs das Wissen produziert oder „prädeterminierend“ darauf einwirkt, weil sonst dasselbe in verschiedenen Diskursen Verschiedenes sein müsste. Werden hier nicht nur Grenzen unter Texten beseitigt, sondern auch unter Diskursen? Was daraus nämlich paradoxerweise in ihrer Identität siegreich hervorzugehen scheint, sind die einzelnen Bedeutungen der Lexeme, die gleichsam diskursimmun werden. Und was hat damit Derrida zu tun?

Manfred Frank stellte seinerzeit sehr ähnliche Fragen und analysierte dabei Derridas Grundbegriffe - wie marque und restance non-présente als die „minimale Identität“", die bei dem französischen Denker die Aufgabe erfüllen, als semiologische Einheit in einem Diskurs eine Zeit zu überdauern und insofern es zu ermöglichen, in verschiedenen Perspektiven über dasselbe zu sprechen bzw. sich auf dasselbe sprachlich zu beziehen, was auch eine minimale Hoffnung auf eine intertextuelle, interdiskursive und intersubjektiv zugängliche Identität eines Gegenstandes/Themas oder einer Bedeutung erweckt.

Franks Interesse an Derrida und anderen (Neo-)Strukturalisten wird dabei durch das Thema der potentiellen Versöhnung des systematischen Charakters von Texten geleitet, der die intersubjektive Verstehbarkeit (den semiotischen Schlüssel und die minimale Identität des Gesagten) verbürge, mit ihrer „Geschichtlichkeit“, die Frank mit der (letzten) „Unausdeutbarkeit“, „Nicht-Identität“ als „Ungleichzeitigkeit der Botschaft“ verbindet.

Im Folgenden wird Derridas Vorstellung „der minimalen Identität“ im Rahmen seines Verstehens von „der offenen Struktur“ oder der „Dezentrierung“ wiedergegeben, durch die er Saussures Strukturtheorie innoviert. Dabei verfolgen wir Franks diesbezügliche Kritik, die mithilfe der von Saussure nachgelassenen Schriften erfolgt. Schließlich bedenken wir das Ergebnis dieser hermeneutischen Kritik und formulieren einige Zweifel.

Derridas Konzeption etablierte sich in der Auseinandersetzung mit Edmund Husserl und Ferdinand de Saussure: in seinen entscheidenden Texten grenzte er sich gegen Husserls Bedeutungstheorie und gegen die Residuen der „metaphysischen“ Tradition in Saussures Denken ab, indem er das (von Saussure vertretene) Prinzip der Differenzialität radikalisierte ${ }^{4}$.

Derrida bewilligt Saussures Vorstellung der Untrennbarkeit von Signifiant und Signifié sowie seine Ablehnung, die Verbindung beider Zeichenmomente mit der Beziehung von Leib und Seele zu vergleichen. Damit habe Saussure nicht nur den bezeichneten Inhalt, sondern auch die Ausdruckssubstanz desubstantiviert, die weder etwas Phonisches noch etwas Materielles ist (Derrida 1993, S. 92). Auf diese Weise habe Saussure den Zeichenbegriff gegen die "metaphysische Tradition“ gewandt ${ }^{5}$.

Auf der anderen Seite liege im Zeichenbegriff als solchem die westliche Metaphysik aufbewahrt und selbst Saussure war nach Derrida nicht imstande, dieser Erbschaft

3 Besonders ausführlich in: Frank 1984, S. 88-102 und 490-571.

4 Und Derrida hat sich so auch verstanden - als ein konsequenter Saussurescher Denker (Derrida 1972, S. 49).

5 Die abendländische Metaphysik - stellt zumindest seit Platon den Glauben an den Bestand einer übersinnlichen Welt dar (oder das Denken aus - übersinnlichen - Prinzipien). Spätestens seit Heidegger wird sie als Beherrschungswissen bzw. -technik dekonstruiert. 
standzuhalten. Das werde schon in dem angenommenen Gegensatz Bezeichnendes Bezeichnetes innerhalb des Zeichens sichtbar sowie in der damit verknüpften Identifikation von Signatum und Begriff, der sich auf diese Weise „potentiell von der Sprache loslöst“ (Derrida 1993a, S. 33). Somit nähert sich Saussure, laut Derrida, der Idee des „transzendentalen Bezeichneten“, einer übersprachlichen Entität, die nicht mehr als Bezeichnendes funktioniert und die Zeichenkette überschreitet.

In diesem Zusammenhang teilt Derrida mit, dass sich das Thema des transzendentalen Signifikats „im Horizont der reinen und eindeutigen Übersetzbarkeit“ konstituierte: es ist eine solche Übersetzung, die den Unterschied zwischen Signifikant und Signifikat geltend macht. Derartige Übersetzung, die „reine“ Signifikate, unberührt und unversehrt, durch gleichsam sterile Signifikanten überträgt, gebe es nicht. Derrida lehnt es sogar ab, von Übersetzung zu sprechen, und schlägt stattdessen einen angeblich angemessenen Ausdruck vor: Transformation des Textes.

Wenn man es zulässt, dass Derrida hier die Saussuresche Idee der Untrennbarkeit oder der gegenseitigen Konstitution von Bezeichnendem und Bezeichneten bloß konsequent anwendet, muss man auch - gegen Derrida - zugeben, dass das Arbitraritätskonzept des Strukturalismus Saussures in dieser Theorie selbst unhaltbar ist: wie könnte die Arbitrarität gedacht werden, wenn das Bezeichnete in seiner Kontur und Identität von dem Bezeichnenden abhängig ist? Insofern ist Derridas Lob für Saussures rein formelles und differentielles Prinzip, das die Arbitrarität mit sich bringen sollte, widersinnig. ${ }^{6} \mathrm{Au}-$ Berdem scheint der Angriff an Übersetzbarkeit selbst nichts anderes als die Ablehnung des Arbitraritätsprinzips zu sein. ${ }^{7}$

Derrida erwidert dem bisherigen Strukturalismus, dass keine formale Identität der Struktur existiert. Damit hängt zusammen, dass es weder ein Strukturzentrum noch mehrere nebeneinander vorkommende Zentren gibt: wir sind in Strukturen verstrickt und haben keine Möglichkeit, hinter unser In-Strukturen-Sein zu gehen ${ }^{8}$, was unter anderem als Einwand gegen Foucault gilt. Die Alternative? Dezentrierung: unendliches Spiel von Differenzen.

Der Prozess des Bezeichnens ist somit das formelle Spiel der Differenzen oder „Spuren“ (Derrida 1993a, S. 38) und Derrida benennt ihn differance. Mit diesen Bezeichnun-

6 „Das Thema der Arbitrarität wird so abgelenkt von seiner versprechenden Richtung (auf die Formalisierung) zu der hierarchisierenden Teleologie“ (Derrida 1993, S. 34).

7 Wenn man mit Derrida Sinn und Wert de facto gleichsetzt, hat man die Arbitrarität aufgegeben. Was ist an einem negativ definierten Wert, einer „Spur“, arbiträr? Indem es nichts bezeichnet, keinen Sinn hat, kann keine Rede davon sein, dass es arbiträr ist. Oder soll es vielleicht einen negativ definierten Punkt, also sich selbst bezeichnen? Und lässt sich dieser Punkt auch durch einen anderen (etwa einen einer anderen „Sprache“ bezeichnen), damit wir uns überzeugen können, dass die Verbindung arbiträr ist? Da es vielmehr nicht arbiträr ist, ist es auch nicht übersetzbar.

8 Damit werde ein Paradox der westlichen Philosophie überhaupt erfasst: ein Prinzip soll einerseits an sich bestehen und von nichts abgeleitet sein, es ist andererseits als Prinzip von etwas, z. B. der sinnlichen Welt, in die Beziehung zu einem anderen eingetreten (vgl. Frank 1984, S. 79-80); wenn bestimmt sein bedeutet nach Scholastikern und Strukturalisten negiert zu werden (negatio est determinatio), dann kann sich nichts außerhalb der Struktur befinden: es kann kein außerstrukturelles und strukturstiftendes Prinzip geben. - Nach unserer Meinung wird hier von Derrida der klassische Fehlschluss gemacht, wo ratio essendi mit ratio cognoscendi verwechselt wird. 
gen meint er ausdrücklich die Struktur und die Bewegung, die Differenzen generiert und dank der die Elemente aufeinander beziehen. Diese Bewegung bildet Lücken, Intervalle, ohne die die vollen Zeichen nichts bezeichnen würden. Derrida hebt es hervor, dass die Differenzen nicht „tombées du ciel“ (Derrida 1993a, S. 39), in keinem geschlossenen System, in keiner statischen Struktur untergebracht sind, die eine synchrone und taxonomische Operation fassen könnte. Da die Bewegung der differance der Präsenz eines Wertes (einem „Transzendentalen Bezeichneten“) nicht unterzuordnen ist, ist ihre Wiedergabe unvereinbar mit dem statischen, synchronen, taxonomischen und ahistorischen Motiv des Strukturbegriffs. Dieser, insofern er als ein endlicher Verweisungszusammenhang zwischen endlich vielen oppositiven Werten zu denken ist, trägt die metaphysische Erbschaft in sich und ist, mitsamt dem Psychologismus, Phonologismus ${ }^{9}$ und Ausschluss der Schrift, durch die Hervorhebung des (Saussureschen) formellen und differentiellen Ansatz zu überwinden (Derrida, 1993a, S. 46; Derrida 1972, S. 49).

Manfred Frank kommentiert vor allem zwei Derridas Behauptungen: dass alles Sinnhafte der Rede im differentiellen Spiel dessen gründe, das selbst nicht den Charakter des Sinnes hat (und das der spätere Derrida marques-non-présentes benennt), und dass dieses Spiel kein in sich geschlossenes System ist, das von einer bestimmten Menge Regeln beherrscht würde, wodurch die Idee der Taxonomie angegriffen ist (vgl. Frank, 1984, S. 102). Und der Kommentar konfrontiert strategisch Derridas Ausführungen mit Saussures Standpunkten, die Derrida konsequent entwickelt haben will.

Frank übersetzt das „taxonomische“ Problem innerhalb der Theorie von Saussures Cours in die folgende Betrachtung: Das Kommunikationsmodell, in dem der Ausdruck, der selbst ein Effekt oppositiver Unterscheidungen von anderen Ausdrücken sei, als bloBes Transport-Instruments vom signifié funktioniert, erläutert nicht, aufgrund welches Faktors die differentielle Wertbestimmung in jeweiligem Fall erfolgt:

Wer wollte ... im voraus absehen, von wie vielen anderen Ausdrücken ich meinen Ausdruck abgrenze? (... .) Die Kette der Negationen (a ist nicht b und nicht c und nicht d usw.) verläuft also möglicherweise im Unendlichen: es liegt letztlich in der interpretatorischen und sprachlichen Kompetenz - ja sogar Phantasie - eines Individuums, welchen Term es auf welche Weise (metaphorisch, metonymisch) verbindet usw. Nichts verlangt also, die Strukturalität der Zeichen-Artikulation an den Gedanken einer in sich geschlossenen Struktur zu binden. Wenn das zutrifft, ist der klassische Strukturalismus unhaltbar geworden, und mit ihm jene Art von Kommunikationstheorie, die darauf setzt, dass ein regelkonform enkodierter Inhalt von einem Sprecher gleicher Kompetenz auf exakt die gleiche Weise dekodiert werden müsse (Frank, 1984, S. 94, Franks Hervorhebung).

Darauf zu beharren, hieße die Realität der Kommunikation zu verleugnen. Das aber anzunehmen, bedeutet, dass man aus der Tatsache der Differentialität der Zeichenbildung auf die Systematizität der Struktur nicht schlussfolgern kann (Frank, 1984, S. 94).

9 Nach Frank hat Saussure jedoch die Stimme nicht wegen der metaphorischen Unsinnlichkeit privilegiert, sondern wegen ihrer Unidimensionalität: nur im Zeitfluss der Rede lassen sich Seme parasemisch voneinander unterscheiden (Frank 1984, S. 92), vgl. unten. 
In diesem Punkt, dem der Unabschließbarkeit der Struktur, sind sich Derrida und Frank einig.

Berührt wird aber bei dieser Auslegung Franks die wesentliche Frage der subjektiven Teilnahme der kommunizierenden Subjekte an der (je) relevanten Konstellation der differentiellen Werte, die die Ausdrücke repräsentieren. Wo Frank ein individuelles Engagement der verstehenden Subjektivität sieht, scheint Derrida eine universelle (Natur?-) Kraft anzunehmen. Also doch ein Fallen vom Himmel.

Die Verfasstheit dieses wirkenden Prinzips Derridas, der différance, widerspricht somit den klassischen Kommunikations- und Zeichenstrukturbegriff. Dies ist möglich, weil der französische Autor die Idee der Unterschiedenheit so radikal auffasst, dass es sich von dem Zeichen nicht mehr sagen lässt, es sei sich unmittelbar gegenwärtig. Während im bisherigen Strukturalismus, etwa bei Lévi-Strauss, die Zeichentransformationen die Struktur unversehrt ließen (vgl. Frank 1984, S. 95), wird diese bei Derrida unstabil, denn die Zeichen verlören ihre herkömmliche „Präsenz“.

Derridas Radikalisierung des Differenzierungsprinzips, die die Zeichen ihrer Gegenwart beraubt, besteht in der Betrachtung, dass wenn ein Zeichen seinen bestimmten Wert oder seine bestimmte „Bedeutung“ nur durch die Unterscheidung von allen anderen erwirbt, es offensichtlich erst dann auf sich selbst verweist, nachdem es den Umweg über alle anderen Zeichen des Systems zurückgelegt hat. Das bedeutet, dass es „von sich ... durch das ganze Universum aller anderen Zeichen getrennt ist“ (Frank 1984, S. 96).

Aus diesen Schilderungen wird sichtbar, dass Derridas Kreuzzug gegen die metaphysische Präsenz zum Konzept einer Art Allgegenwärtigkeit von allem in allem gelangt ${ }^{10}$ und dass er im Unterschied zu Frank keine Instanz der De-limitierung oder je individueller Unterscheidung als sinnbildender Tätigkeit annimmt und annehmen kann. Derridas Naturalismus bezüglich der Ausdifferenzierungsbewegung, der unterstellt, dass sich die Werte und infolge derer sogar die Bedeutungen irgendwie von selbst entfesseln, zeigt sich hier in einer sehr problematischen Gestalt. ${ }^{11}$

Frank selbst ist in diesem Zusammenhang mehr interessiert an der verneinend zu beantwortenden Frage, ob sich ein prinzipielles oder strukturelles Hindernis nennen lässt, das den Differenzierungsprozess der Zeichen innerhalb einer stabilen und identischen Struktur zurückhalten kann, mit der der klassische Strukturalismus Saussures, wie der Terminus „synchron“ oder „langue“ zeigt, zu laborieren scheint.

Frank analysiert die Folgen von Derridas Konzept der différance für Saussures Theorie und im Anschluss an Samuel Weber (1980) konstatiert er einen Widerspruch im Denken des Genfer Linguisten, nämlich die Spannung zwischen der anarchischen und negativen

10 Schon für Buddha stellte das Weltall ein riesiges Netz dar, das aus zahllosen Edelsteinen gestrickt wurde, von denen jeder zahllose Flächen besitzt. Jeder Edelstein spiegelt in sich alle anderen Edelsteine im Netz (Sogyal Rinpoche 1994, S. 50).

11 Wir sehen gerade darin die szientistisch-metaphysische und idealisierende Fiktion: man tut so, als ob die Nummern, Gesetze, Unterschiede, Theorien, Taxonomien, Bedeutungen, Werte unabhängig von den Vollzügen des theoretisierenden Bewusstseins umherschwebten. Aus diesem Standpunkt ist Derrida ein Szientist, der die theoretischen Größen im Unterschied zu klassischen Szientisten mobilisiert hat und ihre Profile durch eine entfesselte Bewegung generieren ließ. 
Macht der Differenzierung und der transparenten Ordnung von Oppositionen als Positivitäten innerhalb eines etablierten Systems ${ }^{12}$ :

Denn wenn beides zugleich gelten soll: erstens, dass es in einer Sprache keinerlei Positivitäten, sondern nur negative Beziehungen gibt, und zweitens, dass die Sprache ein endliches Arsenal von Zeichen ist, dann musste notwendig ein Übergang vom freien Spiel der Differenzen zum fixierten Bezug der Oppositionen gefunden werden. Die Frage ist nur: welche Instanz determiniert das freie Spiel der Relationen? (Frank, 1984, S. 97)

Saussures Antwort, es handle sich um die Einheit eines conscience collective (das an sich amorph und an die Artikulierung dessen angewiesen sei, wessen Ordnung es nun begründen soll), bezeichnet Frank als „heikel“. Saussure spricht schließlich im Cours von einem parallélisme, der die anfängliche Untrennbarkeit zwischen son und pensée zu ersetzen scheint (Saussure 1966, S. 167, vgl. Frank 1984, S. 97). Aus diesem Widerspruch ${ }^{13}$ nimmt Frank nur die Konsequenz, dass „die Grenze zwischen Oppositionen und Differenzen nie endgültig sein kann“ (Frank 1984, S. 98) - als ob die geordneten Oppositionen im Strukturalismus einzig erklärungsbedürftig seien, während die „Differenzen“ als HÝLÉ PROTÉ, wenn auch eine semi-aktive, existierten. Nach unserer Ansicht müssen auch die Werte oder die „distinktiven Züge“ bezüglich ihres „Naturalismus“ untersucht werden.

Da der genannte Agens der Oppositionen nach Saussure selbst kein prinzipieller Garant der Stabilität von langue ist (la loi synchronique est générale, mais elle n'est pas imperative, Saussure 1966, S. 131), hält Frank seine eigene und Derridas These für erwiesen, dass „der radikalisierte Gedanke der Differentialität der Struktur die Idee aufhebt oder doch haltlos macht, es gebe ein außerstrukturales Prinzip, das über ihre Einheit wacht" (Frank 1984, S. 98), bzw. dass „alles möglich gegen die Polizei der Sprache /langage/ ist“ (vgl. Derrida 1988, 72). Es gibt aber nach Saussure, wie wir sahen, ein außerstrukturelles Prinzip, das kollektive Bewusstsein, auch wenn seine anderen Äußerungen damit inkompatibel sind. Das kollektive Bewusstsein verleihe laut Saussure der Struktur deren Einheit, obgleich nicht auf Dauer und obgleich diese Einheit natürlich aufs Bewusstsein, sei dies kollektiv oder individuell, nicht determinierend wirkt. Wäre die Struktur kausal wirkend, könnte das Bewusstsein die Funktion nicht erfüllen, als außerstrukturelles Prinzip der Struktur (je erneute) Einheit zu verleihen. An der zuletzt zitierten Stelle sollte daher von Frank noch hervorgehoben werden, dass sogar nach dem Saussure von Cours zumindest die strukturelle Möglichkeit der Sinnbildung durch das Bewusstsein formuliert ist, die nicht weg-radikalisiert werden kann. Der Konsensus zwischen Frank und Derrida

12 Bei Saussure geht es um diese entgegengesetzten Formulierungen: dans la langue il n'y a que des différences sans termes positifs (Saussure, 1972, S. 166) und Bien que le signifié et le signifiant soient, chacun pris à part, purement différentiels et négatifs, leur combinaison est un fait positif (Saussure, 1972, S. 166).

13 Saussure hat die Bestimmtheit des Sinns aus dem Spiel der Paraseme ausgegrenzt - auch wenn signifiant und signifié nichts Ursprüngliches seien, sondern sie bezögen ihren Unterschied aus der Differenzierung/Artikulation der Werte (Frank 184, S. 91). Frank macht in diesem Zusammenhang allerdings in Richtung Derrida darauf aufmerksam, dass Saussure in seinen nachgelassenen Notes item mit der Möglichkeit arbeitete, auf den zweistelligen Zeichenbegriff zu verzichten und diesen mit einem einstelligen zu ersetzen: er nannte ihn sème und fasste ihn als unmittelbaren Effekt differentieller Beziehungen zu anderen Semen. 
in Bezug auf die Idee der Absenz des Determinismus des Systems (im Sinne dessen bestimmenden Einflusses auf den Sprecher) spielt sich auf dem Boden der Tatsache ab, die gegen den (Neo-)Strukturalismus gerichtet ist und die Frank und Saussure auf die eine („hermeneutische“) Seite stellt, nämlich in der Frage danach, ob die Subjektivität als Effekt des Differenzierungsspieles der Zeichen (marques) aufzufassen ist und neben andere Sinneffekte gestellt werden kann.

Was den Objektpol (wenn man es so ausdrücken kann) der Zeichenbildung bei Derrida betrifft, ersetzte er, wie schon erwähnt, den mit Dualität konnotierten signe durch marque. Diesen uni- und zugleich pan-lateralen Zeichenbegriff mit anscheinend keiner Stabilität erläuterte der Autor in (Derrida 1988, S. 51), auch unter dem Eindruck von der Kritik, die darauf verwiesen hatte, die Strukturalität ließe sich derart nicht verabsolutieren, da jede Abweichung oder Transformation (der Zeichen) eine zu erkennende Identität voraussetzt, von der abgewichen wird: jedes Unterscheiden setzt einen Unterscheidungsgrund voraus, der den unterschiedlichen Elementen gemeinsam ist. An der angegebenen Stelle antwortet Derrida seinem Diskussionspartner, John Searle, auf den Einwand mit seinem Neologismus restance non-presente, bzw. non-present remainder (Franks Übersetzung: die nicht-selbstgegenwärtige Bleibe). Der Ausdruck ist gegen die Vorstellung einer dauerhaften Präsenz eines Zeichens oder einer Bedeutung gerichtet: die Bedingung des Abweichen-Könnens darf keine permanence sein. Was in der Veränderung verbleibt, sind non-present remains, wie Derrida Searle erklärt:

Die Wiederholbarkeit eines Elementes entzweit a priori seine eigene Identität, auch abgesehen davon, dass sich diese Identität nur durch differentielle Relationen zu anderen Elementen bestimmen oder abgrenzen kann und durch diese Differenz gekennzeichnet ist. Weil die Wiederholbarkeit innerhalb von jedem Element und unter den Elementen gegenseitig der differentiellen Natur ist, weil sie jedes Element konstituiert, indem sie ihn entzweibricht, weil sie ihn mit einer Artikulationsspalte gekennzeichnet, ist diese(r) Rest/Resistenz (restance, remainder), wie auch immer unentbehrlich, niemals ein Beharren der vollen Präsenz: es ist eine differentielle Struktur, die der Präsenzlogik sowie der Opposition (sei diese einfach oder dialektisch) der Präsenz und der Absenz entgleitet, der Opposition, von der die Idee der Permanenz abhängt. Das ist der Grund, warum die marque als nicht-präsente Resistenz kein Gegensatz zu der Abschaffung der marque ist. Ebenso wie die Spur ist die marque weder präsent noch absent (Derrida 1988, S. 53, meine Übersetzung). ${ }^{14}$

14 The iterability of an element divides its own identity a priori, even without taking into account the fact that this identity can only determine or delimit itself through differential relations to other elements and that it hence bears the mark of this difference. It is because this iterability is differential, within each individual „element" as well as between the „elements,“ because it splits each element while constituting it, because it marks it with an articulatory break, that the remainder, although indispensable, is never that of a full or fulfilling presence: it is a differential structure escaping the logic of presence or the (simple or dialectical) opposition of presence and absence, upon which opposition the idea of permanence depends. This is why the mark qua „non-present remainder" is not the contrary of the mark as effacement. Like the trace it is, the mark is neither present nor absent (Derrida 1988, S. 53). 
Fragen wir nun, was diese restance non-présente, die Frank an anderer Stelle mit Saussures Termin aposème analogisierte, eigentlich bedeutet. Ist sie bezeichnend oder Spurentragend an sich? Ist es eine physikalische Entität? Der von Saussure abgelehnte naturalistische Fehlschluss lautete, dass Naturlaute an sich genommen keine Bedeutungsträger seien, sie werden es, nachdem man sie als signifiant interpretiert hat. Dies ist Frank zufolge nur verstehbar, wenn man das hypothetische Urteil (Abduktion) einbezieht und dafür verantwortlich macht („keine Identifikation ohne Signifikanz!“, sagt Frank /1984, S. 551/). Der Sinn ist nicht „nezessitiert von der Naturseite der Töne, Spuren und Konexionen“, er kann nicht von selbst aus der marque bzw. aus den Beziehungen generiert sein, die sie zu anderen marques unterhält, sondern er wird durch „eine freie interpretatorische Zusprechung" konstituiert:

Um auch nur vage als ,bleibende Marke“ charakterisiert werden zu können, muss der Marke ein Sinn zugeordnet sein. Wenn aber diese Sinn-Zuordnung die Bedingung ist, unter der ich überhaupt erst von der Einheit der Marke sprechen kann, hieße es, einer petitio principii sich schuldig zu machen, wenn man den Sinn einerseits als von der ,Wirkung der Marke determiniert ausgeben wollte. Um diese sinndeterminierende Wirkung ausüben zu können, müsste die Marke - vor allem Sinn schon einheitlich verfasst sein. Was aber wäre eine Marke vor allem Sinn anderes als ein Naturding? Naturdinge als , an sich distinkt' annehmen hieße, der ,illusion naturaliste‘ zum Opfer fallen, deren entschiedene Ablehnung allererst das Feld eröffnet, auf dem eine strukturalistische und neostrukturalistische Zeichentheorie möglich ist (Frank 551-552).

Wenn man Derridas Formulierungen gefällig liest und die restance non-présente nicht als Negation jeder, sondern nur der absoluten und dauerhaften Identität auslegt, ließe sich der Begriff Frank zufolge sinngemäß Humboldts Diktum annähern, der Sinn eines Zeichens habe keine bleibende Stätte in der Schrift, oder damit „so etwas“ meinen „wie die schematische Einheit eines Zeichens, von der Schleiermacher sagte, sie sei eine Einheit mit verschiebbaren Rändern, die auch den vermeintlichen Sinn-Kern auslöschen oder neu festsetzen kann“ (Frank 1984, S. 99). Bei Derrida findet Frank diese Idee mit dem Mechanismus beschrieben, wie das Transformieren der Einheit eines Zeichens erfolgt: „... die Differenzierung spalte nicht nur das Zeichen (qua Oppositum) vom anderen, sondern spalte - kraft der unabweisbaren Möglichkeit der Wiederholung - bereits die Einheit dieses Zeichens selbst" (Frank 1984, S. 100). In der Zeitlichkeit des Zeichens liegt in Franks Lesart Derridas also der Differenzierungsprozess (Derridas différance), die Tatsache, dass Zeichen nicht nur von anderen in oppositiven Relationen untereinander getrennt sind, sondern dass jedes Zeichen von sich im Zeitfluss so abgespalten ist, dass „die von ihrer ... materiellen Fracht befreite Idealität eines Zeichen-Sinns aufleuchte/t/“ (Frank 1984, S. 101). Zwei Werte können nur in der Zeit unterschieden werden und die Zeichenmaterie muss zuerst in die Vergangenheit versunken sein, um ihrer Bedeutung freien Raum zu bieten, meint Frank.

Derridas Ansatz widerspruchslos angelegt und zu Ende gedacht sähe in concreto folgendermaßen aus: „Eine Bleibe wäre es, insofern ein und dasselbe aposème /hypothetisch 
dasselbe - meine Bemerkung/ nacheinander beliebig vielen hypothetischen Sinnzuschreibungen offen stünde" und „/n/on-présente wäre die Kette, indem keine Einschreibung sich in der ihr folgenden sinngleich und gleichförmig fortsetzen muss, derart, dass die Suipräsenz ihres Bedeutens eine von keiner Differenz getrübte instantane Einheit bildete" (Frank 1984, S. 553). Das heißt: jeder Zeichengebrauch beruht auf einer Hypothese. Diese ist als motivierende Kraft für die folgende Hypothese bzw. für den nächsten Zeichengebrauch zu denken. Zwischen den einander folgenden Hypothesen bzw. Zeichengebräuchen bzw. Interpretationen besteht eine Kontinuität - die „von einander motivierenden hypothetischen Urteilen“ (Frank 1984, S. 553).

Auf diese Weise wird dem naturalistischen Fehlschluss entwichen, Naturursache verwandelt sich in Grund, der die Handlung (die Sinnerzeugung) nie auslöst, sondern veranlasst - motiviert. (Der Grund motiviert erst, sobald es als solcher anerkannt bzw. interpretiert ist ${ }^{15} /$ vgl. Frank 1984, S. 554/).

Was also die von Derrida intendierte Aufgabe der différance besser als diese selbst ausüben könne, sei die einzig motivierbare, urteilende und die „Nicht-Koinzidenz der Zeichensynthesis“ bewirkende selbstbewusste Individualität (vgl. Frank 1984, S. 555). Sie erzeugt die Reihe der „unabsehbaren (Neu-)Interpretationen“ schon interpretierter Marken, die „in keiner authentischen Anschauung instantan“ repräsentierbar oder vergegenwärtigbar ist (Frank 1984, S. 556).

Das sinnfähige individuelle Bewusstsein ist als „freie Überschreitung“ ein Gegenstück zu dem virtuell stabilisierenden System von grammatischen, semantischen und pragmatischen Regeln. Sie erwirkt, dass der jetzige Sinn nicht aus ihnen ableitbar ist, dass zu der jeweiligen Bedeutung nicht unter den methodisch kontrollierbaren Verfahren aus der Signifikation gelangt werden kann, „die das betreffende Zeichen bis dahin innehalte“. „Keine semantische Deduktion aus präetablierten Prämissen“ findet im Sprachverstehen statt, denn

„das individuelle Moment ... liegt in dem Abstand, den es zwischen der alten und der neuen Bedeutung gehöhlt hat. In diesem Sinne ist Verstehen re-produktiv: Nachvollzug nicht der ehemaligen Bedeutung, sondern des Sinnentwurfs, der die alte Bedeutung im Entwurf einer neuen des-aktualisiert, ent-gegenwärtigt und irremediabel aufspaltet“ (Frank 1984, S. 557).

Wie soll man sich nun aber, so fragen wir, den aufspaltenden Zugriff vorstellen? Wie beziehe ich mich per das aposème auf die vorangehende Interpretation? Was sagt mir an dem aposème, wie er bislang hypothetisch ausgelegt wurde? Ein Aposem ist Frank zufolge kein Grund, der mich motivieren kann; es soll die fremde Hypothese sein. Wie soll aber diese neu zu interpretierende vorausgehende und aufzuspaltende Interpretation

15 Franks Ansatz ist hier folgerichtig und weitgreifend, selbst die Naturwissenschaften werden nicht geschont: „auch physische Ursachen sind letztlich Gründe in dem Sinne, dass die Seinsweise des Physischen durch Wahrnehmungsurteile (und mithin durch Interpretationen) als das, was sie ist, erschlossen ist: Auch die Gesetze der Mechanik sind nichts als motivierte Schlussfolgerungen am Leitfaden von Wahrnehmungsurteilen, deren hypothetischer und also hermeneutischer Charakter niemals zu überwinden ist und auch nicht überwunden werden soll, weil mit ihm die Verständlichkeit eben der Welt, in welcher wir mechanische Gesetzmäßigkeiten am Werke sehen, verschwinden würde" (Frank 1984, S. 555). 
im individuellen Bewusstsein des nun Interpretierenden „aufgefasst“ werden? Muss ich selbst sie - als fremde - erstellen? Motiviere ich mich von selbst aufgrund meiner älteren zu einer anderen, wiederum meiner Hypothese?

Frank erklärt, dass die neue Bedeutung aus der herkömmlichen nicht abgeleitet werden kann, dass sie sie nur frei motiviert (Frank 1984, S. 556). Muss ich die alte aber zuerst nicht synthetisieren, damit ich mich von ihr beeinflussen lassen kann? Sie ist nicht gegeben. Das Aposem selbst muss als solcher interpretiert werden (als aposème einer signifiant-signifié-Synthesis, von selbst, naturgemäß ist es eigentlich als ein Empirisch-Materielles kein aposème). Dann motiviere ich mich durch meine eigene vorherige Interpretation? Die Frage nach der Verfasstheit des Feldes der semantischen Kontinuität wird ohne den Rekurs auf den Begriff des kollektiven Bewusstseins wohl noch viele Schwierigkeiten bereiten.

Beim Begründen dessen, dass ein in der Rede wiederholtes Zeichen seine Identität nicht garantieren kann, schildert Derrida (mit Franks Zustimmung), wie die Elemente einer überindividuellen Botschaft, also die grammatischen, semantischen oder pragmatischen Typen, daran beteiligt sind, dass der individuelle Sinn zurücktritt, sodass ein freier Raum für die neue individuelle Auffassung zustande kommt: wenn man sich äußert, verliert man die Kontrolle über das Geäußerte (es ist sozusagen dem konventionellen Typ ausgegeben), sodass diesem ein anderer individueller Sinn zugeschrieben werden kann und muss. Das Überindividuelle Allgemeine, der konventionelle Sinn, scheint in dieser Konzeption Derridas eine öffentlich zugängliche Opferstätte zu sein, wo das Individuelle dargebracht und verlassen wird, um von einer anderen individuellen Deutung übernommen zu werden. Der Sinn wird auf diese Weise durch die Wiederholung je neu gekennzeichnet (re-marque), so dass man rhetorisch fragen kann: „Wer beweist, dass der Sinn eines Zeichens nach dem Durchgang durch die Lücke der Iteration in derselben Synthesis mit seinem Ausdruckssubstrat steht wie zu Beginn?" (Frank 1984, S. 542; vgl. Derrida, 1988, S. 61) Die Voraussetzung dessen kennen wir nach Derridas und Franks Auslegungen schon: keine Kopräsenz von Sprecher und Hörer und keine Synchronie von Zeichen und dessen Sinn. Die Ungleichzeitigkeit der letzteren bedeutet, dass das zu Interpretierende - der allgemeine Sinn oder der Sinn eines anderen - nicht gegeben ist. Wie kann nun aber, so fragen wir, der individuelle Sinn zurücktreten zu Gunsten vom Überindividuellen, das nie gegeben ist? Was wird also gespalten und was überdauert als minimale Identität innerhalb von mehreren Interpretationen bzw. Sinnzuschreibungen? Wird das Aposem - in der Rolle des überindividuellen - durch die Interpretation „gespalten"? Ein Aposem, das an der Zeichenbildung überhaupt nicht teilnehme? Auch wenn es als solches nur durch Beziehung auf das ihm zugehörige signe ist?

Wenn Jacques Lacan, dessen Auslegungen zum disseminalen Charakter des Zeichens Derrida stark beeinflusst haben (Frank 1984, S. 544), über das stetige Gleiten des signifié unter dem signifiant spricht (vgl. Lacan 1957, Vortrag) wird er von Frank berichtigt, unter dem Signifiant könne der Signifikat nicht gleiten. ${ }^{16}$ Nach Saussure könne sich doch das Zeichen nur als Ganzes verändern, weshalb er später eben den Begriff ( $a p o)$

16 Die kritisierte Formulierung Lacans: glissement incessant du signifié sous le signifiant (Lacan 1957, Vortrag). 
sème eingeführt hätte. Aposème heiße: eine Art „Worthülse, materiell-empirische Verkörperung des Zeichens, die selbst in die Bezeichnung bzw. Semiosis nicht eingeht" (Frank 1984, S. 544). Dann ist ein Aposem das, was bestehen kann, während sich das Zeichen semantisch ändert.

Bei Lacan scheint das Überindividuelle (des Zeichens), das den individuellen Zeichengebrauch einer von ihm nicht kontrollierbaren Rezeption (durch eine neue individuelle Hypothese/Interpretation) aussetzt und das ein Intersubjektiv-Objektives bedeuten könnte, einerseits in der Zukunftsoffenheit jeder Bedeutungsverleihung und andererseits im sozialen und kontextuellen Gedächtnis der Zeichen zu liegen (Lacan 1957, Vortrag; vgl. Frank 1984, S. 546). Zeichen enthalten nämlich in sich die Spuren ihrer vergangenen Verwendungen und diese wirken mitbestimmend im Rahmen der „synchronen“ parasemischen Beziehungen. Je einzelnes Anwenden von Zeichen, das immer dialogisch sei (und deshalb auch nach Lacan und Frank nie monopolisierbar ${ }^{17}$ ), könnte dann in einer je individuellen Verengung oder Reduktion der virtuellen Menge von vergangenen und künftigen Sinnmomenten beruhen. Frank jedenfalls, wie schon erwähnt, scheint in diese Richtung zu denken: Lacans Metapher von am Zeichen „vertikal“18 angelagerten vergangenen Kontexten (und den Antizipationen von Sinn) verknüpft er mit Schleiermachers Rede von der Interpretation als „eine/r/ unendliche/n/ Aufgabe, weil es ein Unendliches der Vergangenheit und der Zukunft ist, was wir in der Rede sehen wollen“ (Schleiermacher 1977, S. 94). Und in diesem Zusammenhang schreibt er: „Denn niemals ist vorhinein abzusehen, welche Gruppe von parasèmes die Semantik eines Zeichens oder einer Zeichenkette in einer historisch-einmaligen Kontextkonstellation tatsächlich determinieren wird“ (Frank 1984, S. 545).

Aus den Formulierungen sieht man besser, wie die Intersubjektivität an Sinnverschiebungen beteiligt sei, als wie die Intersubjektivität selbst mit zu ihr gehörendem einheitlichem Sinnkontinuum möglich ist. Mehr als um eigene Kohärenz geht es Frank offenbar um Darstellen dessen, dass seine Berichtigung Derridas mit dem ursprünglichen strukturalistischen Ansatz, der bei Saussure zu finden ist, übereinstimmt.

Frank zeigt es an einigen Stellen aus nachgelassenen Texten des Genfer Linguisten, wie dieser zum Thema Arbitrarität:

Was hier den Philosophen und Logikern entgangen ist, ist, dass wenn ein Zeichensystem von den bezeichneten Objekten unabhängig ist, würde es durch die Tatsache der Zeit den Verschiebungen ausgesetzt sein, die der Logiker nicht vorhersagen $\mathrm{kann}^{19}$ /meine Übersetzung/.

Der authentische Saussure bestreitet hiermit die Identität der Bedeutungen zwischen Sprecher und Hörer, spricht des Weiteren ebenso wie Lacan über un certain flottement zwischen dem, „was die Präskripte des Sprachsystems verlangen, und dem, was der

17 „Der Sinn einer Aussage ist immer derjenige, den der Andere damit meint“ (Lacan 1957, Vortrag).

18 Lacan 1957, Vortrag

19 Saussure (1974, S. 13): „ce qui a échappé ici aux philosophes et aux logiciens, c'est que du moment qu'un système des symboles est indépendant des objets désignés, il était sujet à subir, pour sa part, par le fait u temps, des déplacement non calculables pour le logicien." (vgl. Frank 1984, S. 542). 
Initiative des sprechenden Individuums überlassen ist“ (Frank 1984, S. 558; Saussure 1968, S. 277) und außer der zeitlich linearen Sukzession, die seine selbstnominierten Nachfolger ${ }^{20}$ Frank zufolge ignorieren, einen „gegenstrebigen Verallgemeinerungs- oder Idealisierungsprozesses“ annimmt ${ }^{21}$ (Frank 1984, S. 561), der bei Derrida fehlt. Diese mit der kantischen „Synthese der Rekognition“ zu vergleichende Aktivität hat die Funktion, das „vergangene Element und den Ort seines Auftretens im Kontext anderer Elemente im Gedächtnis“ festzuhalten (Frank 1984, S. 561). Und in diesem Zusammenhang berührt Frank konkreter unsere Frage nach der Seinsweise des „Identischen“ im Fluss der wechselnden und zugleich aneinander anknüpfenden Zeichenverwendungen bzw. Interpretationen: was in der Strömung der immer neuen Zeichenverwendungen

„festgehalten wird, kann nun freilich nicht das Element /x/ - bzw. die Elementenkonfiguration (Gestalt) - selbst sein (die sind ja vergangen), sondern nur ihr Stellvertreter: nennen wie ihn ... $\mathrm{x}^{\prime}$. Die unterscheidende Beziehung findet also nicht, wie das Code-Modell voreilig unterstellt, zwischen den Elementen $\mathrm{x}$ und $\mathrm{x}$ statt, sondern zwischen den Elementen $\mathrm{x}$ und $\mathrm{x}^{\prime}$, zwischen einem vergangen-(v)erinnerten und gegenwärtig-vernommenen und nun als $\mathrm{x}$ interpretierten Element“ (Frank 1984, S. 561) ${ }^{22}$.

Daraus ist nach Frank zu folgern, dass der sprachliche Wert nicht einer „zeitlos-synchronen Struktur von Elementen“ zuzurechnen ist, sondern dass er die Idee der Zeit einschließt (Frank 1984, S. 561): Die Zeit schafft die Differenz, die die individuelle Interpretation erst danach hypothetisch als Beziehung zwischen Identischem auslegen kann, es gibt in der Sprache keine Identität ohne Zeit (und ohne Subjekt).

Aus dieser strukturalistisch-hermeneutischen Synthesis, die hier, Lacan, Saussure und Frank vorstellen, tritt stets eine schwer zu beantwortende Frage auf, die das SolipsismusProblem betrifft: Wie absorbiert die vom jeweiligen Subjekt generierte Einheit des Sinnes in ihrem Motiviert-Sein die von anderen Subjekten erzeugten Bedeutungsauslegungen, damit man die Idee des kontinuierlichen und intersubjektiven Semiosis-Prozesses denken kann? Kann die minimale Identität, die eine Zeit überdauert und die Intersubjektivität ermöglicht, selbst erst ein Produkt der synthetischen Tätigkeit (der individuellen Interpretation) sein? Warum ist sie „minimal“, wenn sie je schon holistisch charakterisiert wird (wenn Frank zufolge aus einem Sinnlich-Materiellen durch eine Hypothese ein Aposem entsteht, ist dies Aposem immer eines von einem sème, also dem ehemaligen signifié-signifiant-Komplex)? Das Identische als Substrat der (pluralfähigen) Sinnzuschreibung (oder genauer der Sinnzuschreibbarkeit) soll vielmehr eine Neutralität hinsichtlich der einzelnen möglichen Sinne aufweisen. Ein bestimmtes Aposem erfüllt diese Anforderung nicht und ein materiell-sinnliches Chaos habe in der Semiosis überhaupt keine Rolle.

20 Allerdings zumindest mit Derridas und Lacans Ausnahme.

21 „memorisation“, „recollection des unités phonatoires successives“ (Saussure 1974, N 15; zitiert nach: Frank 1984, S. 561)

22 Frank stützt sich hier auf Christian Stetters Peirce und Saussure, in: Kodikas/Code. An International Journal of Semiotics 2, 1979, 124-149. 
Wenn die offene Struktur die Transformationskontinuität via (minimale) Identität der Elemente nicht garantieren kann, worin sich Derrida und Frank einig sind, dann könnte man sich - erstens - an die Weise erinnern, wie die an der Vorstellung des (geschlossenen) Systems orientierte Sprachphilosophie mit der Frage umgeht, welche sprachlichen - Prinzipien es sind, die es mehreren Subjekten erlauben, in verschiedenen Perspektiven über dasselbe zu sprechen bzw. sich auf dasselbe sprachlich zu beziehen. Frank setzt sich mit den sprachanalytischen Denkern, vor allem mit Peter Strawson und Ernst Tugendhat, in seiner Unhintergehbarkeit (1986) auseinander. Das Problem trägt hier den Namen der Beziehung zwischen einer „epistemischen Asymmetrie“ und der angenommenen systembedingten "grammatischen Symmetrie“.

Nicht weniger anregend - zweitens - sind Husserls Analysen zur Beziehung von Ausdruck-Anzeichen und von Bedeutungsintention - Bedeutungserfüllung der Logischen Untersuchungen, wo ja auch die Möglichkeiten der Identifizierbarkeit von je verschieden (inter)subjektiv zugänglichem Gedachtem und Wahrgenommenem zu Thema werden, sowie Derridas Kommentare bezüglich dessen in La voix et le phénomène. Und - drittens - wäre es wünschenswert, Franks neueste Ansichten der Subjektivität und seine Vorträge zu Präreflexives Selbstbewusstsein zu berücksichtigen, in denen im Zusammenhang mit den Untersuchungen zu neueren Bewusstseinstheorien und im Anschluss an Sartre unmittelbare und opake Inhalte unterschieden werden, die ebenfalls als semiologische Kategorien vorgestellt werden müssen: Von Sartre stammende konzeptuelle Dyade reflet - reflétant, die Idee des transparenten und präreflexiven Bewusstseins sowie der korrelative externalistische Begriff der Gegenständlichkeit bilden das theoretische Milieu einer zukünftigen und hoffnungsvollen Zeichentheorie (vgl. z. B. Frank 2015, S. 168-172). ${ }^{23}$

Bislang scheint Teuberts und Busses diskurssemantischer Streit über das interdiskursiv Zugängliche in einem aporetischen Zustand zu verharren. Der allererste Grund dessen besteht darin, dass man programmatisch über keine Bewusstseinstheorie verfügt, die es erst ermöglichen würde, das naturalistische Feld zu verlassen. Dazu ist nämlich notwendig, auf den widerspruchsvollen Begriff des Diskurses zu verzichten, der einerseits allen Sinn generierende Entität darstellt und andererseits virtuell und vom erforschenden Diskursanalytiker, der selbst als Subjekt für ein Diskursprodukt gehalten werden müsste, konstruiert ist.

\section{Literaturverzeichnis}

BUSSE, Dietrich und TEUBERT, Wolfgang (Hg.) (2013): Linguistische Diskursanalyse: Neue Perspektiven. Wiesbaden: Springer VS.

23 In jedem Fall wäre es eine Illusion zu glauben, es ließen sich grundlegende semiologische oder linguistische Kategorien ohne entsprechende Bewusstseinstheorie beschreiben. Selbst Physik laboriert heute mit Bewusstsein als unvermeidlichem Grundbegriff, man sollte schließlich die Humanwissenschaften nicht naturwissenschaftlicher werden lassen als die Naturwissenschaften selbst. 
BUSSE, Dietrich (2013): Diskurs - Sprache - Gesellschaftliches Wissen. Perspektiven einer Diskursanalyse nach Foucault im Rahmen einer Linguistischen Epistemologie, in: Linguistische Diskursanalyse: Neue Perspektiven Wiesbaden: Springer VS, S. 147-185.

DERRIDA, Jacques (1972): Positions. Entretiens avec Henri Ronse, Julia Kristeva, Jean-Louis Houdebine, Guy Scarpetta, Paris: Minuit.

DERRIDA, Jacques (1988): „Limited Inc abc ..., in: Limited Inc. Northwestern University Press Evanston, IL.

DERRIDA, Jacques (1993a): Sémiologie a grammatologie, in: Texty k dekonstrukci, S. 31-47, Bratislava.

DERRIDA, Jacques (1993b): Hlas a fenomén, in: Texty $k$ dekonstrukci, S. 49-145, Bratislava.

FRANK, Manfred (1986): Die Unhintergehbarkeit von Individualität, Frankfurt am Main: Suhrkamp Verlag.

FRANK, Manfred (1993): Selbstbewusstseinstheorien. Frankfurt: Suhrkamp Verlag.

FRANK, Manfred (1984): Was ist Neostrukturalismus?, Suhrkamp Verlag.

FRANK, Manfred (2001): Sind Bewusstsein und Denken wesentlich sprachlich? Die Abkehr von der ,linguistic turn' in neuerer Sprachphilosophie, in: Wissenschaft, Bildung, Politik. Herausgegeben von der österreichischen Forschungsgemeinschaft, Band 5: Der Mensch und seine Sprache(n), Wien, Köln, Weimar: Böhlau Verlag, S. 109-130.

FRANK, Manfred (2015): Präreflexives Selbstbewusstsein, VierVorlesungen, Stuttgart: Reclam.

LACAN, Jacques (1957): L'instance de la lettre dans l'inconscient ou la raison depuis Freud (prononcé à Paris le 9 mai 1957 devant le Groupe de philosophie de la Fédération des étudiants ès lettres Sorbonne; verfügbar unter http://aejcpp.free.fr/lacan/1957-05-09.htm [zuletzt geprüft am 10. 03. 2017].

SAUSSURE, Ferdinand (1966): Cours de linguistique générale, publié par Charles Bally et Albert Sechehaye, Paris.

SAUSSURE, Ferdinand (1972): Cours de linguistique générale, publié par Charles Bally et Albert Sechehaye, Paris: Payot.

SAUSSURE, Ferdinand (1968 + 1974): Cours de linguistique générale, édition critique par Rudolf Engler, 2 Bände, 1968, 1974 Wiesbaden.

SCHLEIERMACHER Friedrich (1977): F.D.E. Schleiermacher: Hermenutik und Kritik. Hrsg. v. Manfred Frank, Frankfurt a.M.

SOGYAL Rinpoche (1994): Tibetan Book Of Living And Dying, New York: Harperone.

TEUBERT, Wolfgang (2013): Die Wirklichkeit des Diskurses, in: Linguistische Diskursanalyse: Neue Perspektiven, Wiesbaden: Springer VS, S. 55-146.

WEBER, Samuel (1980): Closure and Exclusion, in: Diacritics, June 1980, John Hopkins University Press, S. 35-46.

Mgr. Michal Rubáš / MichalRubas@seznam.cz

Univerzita Palackého v Olomouci, Filozofická fakulta, Katedra germanistiky

Křížkovského 10, 77180 Olomouc, CZ 
